\title{
Plan de mejoras para optimizar el sistema de cobranza de la empresa grupo Vitasana, C.A.
}

\author{
Improvement plan to optimize the collection system of the company Grupo Vitasana, C.A.
}

Plano de melhorias para otimizar o sistema de cobrança da empresa Grupo Vitasana, C.A

《 Alba González

albismg19@gmail.com

ORCID: 0000-0002-5525-6172

Universidad de Carabobo, Venezuela
《 Edixón López

edixonlopez@hotmail.com

0000-0002-3354-6670

Universidad de Carabobo, Venezuela

Recibido: enero 2019 / Aceptado: febrero 2019 / Publicado: mayo 2019

\section{RESUMEN}

El propósito de esta investigación es desarrollar un plan óptimo para el proceso de facturación que beneficie al sistema de cobranza del Grupo Vitasana. La investigación está enmarcada en un Proyecto factible, el tipo de investigación es documental y de campo, con una población y muestra de 9 trabajadores del departamento de facturación, se aplicó una encuesta con preguntas dicotómica previamente validada por expertos. Los resultados obtenidos reflejan que existe deficiencia en la organización y planificación, falta de normas y procedimientos, lo que hace factible la implementación de un plan de mejoras al proceso de facturación por parte de los trabajadores. Lo que permitirá tener un buen control, sobre todos los ingresos diarios, y poder emitir las facturas a tiempo así agilizar su envió al destinatario en este caso (compañía de seguros, empresas) lo cual generara un buen sistema de cobranza en un tiempo real y oportuno.

Palabras clave: Cobranzas; estrategias; facturación; proceso; PYMES, Sistema

\section{ABSTRACT}

The purpose of this research is to develop an optimal plan for the billing process that benefits the Vitasana Group's collection system. The research is framed in a feasible project, the type of research is documentary and field, with a population and sample of 9 workers from the billing department, a survey with dichotomous questions previously validated by experts was applied. The results obtained reflect that there is a deficiency in the organization and planning, lack of standards and procedures, which makes feasible the implementation of a plan to improve the billing process by the workers. This will allow you to have good control over all daily income, and to be able to issue invoices on time, thus expediting your delivery to the recipient in this case (insurance company, companies), which will generate a good collection system in real time and timely.

Key words: Collections; strategies; billing; process; SMEs, System
RESUMO

O objetivo desta pesquisa é desenvolver um plano ideal para o processo de cobrança que beneficie o sistema de cobrança do Grupo Vitasana. A pesquisa está enquadrada em um projeto viável, o tipo de pesquisa é documental e de campo, com uma população e amostra de 9 trabalhadores do departamento de cobrança, foi aplicada uma pesquisa com perguntas dicotômicas previamente validadas por especialistas. Os resultados obtidos refletem que há uma deficiência na organização e no planejamento, falta de normas e procedimentos, o que viabiliza a implementação de um plano para melhorar o processo de cobrança pelos trabalhadores. Isso permitirá que você tenha um bom controle sobre toda a renda diária e possa emitir faturas no prazo, agilizando a entrega ao destinatário neste caso (companhia de seguros, empresas), que gerará um bom sistema de cobrança em tempo real e oportuna.

Palavras chave: Cobranças; estratégias; faturamento; processo; PMEs, Sistema 


\section{INTRODUCCIÓN}

A ctualmente, el mundo de los negocios o empresas debido al proceso de globalización presenta un nuevo panorama, reflejándose en cambios a nivel financiero, económico y tecnológico. En tal sentido surge la necesidad de revisar y mejorar las operaciones que se efectúan en las organizaciones para obtener mejores resultados y convertirse en empresas sólidas. Desde esta perspectiva, las compañías necesitan estar a la vanguardia en el conocimiento de herramientas gerenciales que les ayude a mejorar su capacidad y desempeño basado en la toma de decisiones.

Las empresas hoy en día, han sido afectadas por los desequilibrios económicos que se han generado las últimas dos décadas, dado los elevados índices de inflación y los cambios de política en Venezuela; todo esto afecta los costos operativos e influye en los precios de los productos o servicios que comercializan; igualmente esta situación repercute en la capacidad de compra de los clientes.

Ante esta situación, se requiere que las organizaciones mantengan un sistema dinámico para el logro de las metas organizacionales; siendo el proceso administrativo el que permite guiar la gestión empresarial hacia los objetivos planteados y su posterior evaluación. Al respecto Terry (2007), señala que: "Se trata de la aplicación de la planeación, organización, ejecución y control por medio de los cuales se administra un gerente" (p. 76).

De acuerdo a lo mencionado anteriormente, son importantes los procesos administrativos y contables en la organización, ya que representan un elemento primordial en el desarrollo de las actividades y el uso de recursos en forma eficiente. Sobre estas consideraciones, para asegurar el cumplimiento de los propósitos se hace necesario el establecimiento de estrategias y procedimientos en los procesos de gestión; más específicamente en el de facturación y cobranza.

Se puede señalar, que mediante la facturación se materializa y documentan los ingresos de la organización. Este ciclo se inicia con las cotizaciones solicitadas por los clientes, donde luego se origina un presupuesto formal, posteriormente la venta (prestación del servicio) y nace la cuenta por cobrar. Por lo tanto, las estrategias de negocios deben fundamentarse en un proceso integral con el fin de analizar, observar y verificar como se están llevando a cabo en las actividades de esta área de estudio.

De allí, la importancia de mejorar los procesos, entendiendo por mejoras lo que define Harrigton (2007), en el sentido que "mejorar un proceso significa cambiarlo para hacerlo más efectivo, eficiente y adaptable, qué cambiar y cómo cambiar depende del enfoque especifico del empresario y del proceso" (p. 56).

Por otra parte, dentro del conjunto de actividades que se desarrollan en una empresa uno de los aspectos de mayor relevancia, sin restar importancia a los demás, es lo referente a la política de 
crédito y cobranza por lo que todos los esfuerzos de la directiva deberían estar encaminados hacia el desarrollo de un eficaz proceso de cobro.

De acuerdo a lo mencionado, Hurtado (2006):

La planificación y control de crédito y cobranzas ofrece grandes ventajas al administrador, pues este le dará las pautas a seguir para determinar la situación financiera de una determinada empresa, sirviéndole de fundamento para la toma de decisiones que afecten su patrimonio (p.23).

En Venezuela, según el Instituto de Estudios Superiores de Administración (IESA ), de acuerdo a informes económicos señala, que existen organizaciones que con su amplio crecimiento, obviaron mejorar sus sistemas de información contable y administrativa, generando consigo informaciones contables no adaptadas a la realidad.

Dentro de lo planteado, se presenta la investigación específicamente en la empresa Grupo Vitasana C.A dicha organización nace a mediados del 2008 en la ciudad de Valencia estado Carabobo, Venezuela, dedicándose a la prestación del servicio en el área de salud siendo su principal actividad, la salud ocupacional.

No transcurrió mucho tiempo para que la organización contara con una cartera de clientes en el mercado, en la actualidad tiene afiliada varias compañías a la cual le presta sus servicios (medicina ocupacional) mediante la utilización de empresas aseguradoras las cuales son las encargadas de cancelar lo que cada trabajador se realice o personas particulares que acuden mediante su seguro a utilizar los servicios de salud. Sin embargo en los últimos dos años se ha observado con preocupación, los niveles de rentabilidad y productividad.

De igual forma, presenta una serie de deficiencias en el área administrativa y financiera, las cuales son originadas y detectadas en el momento que se realiza el cierre mensual de la empresa, donde se detalla que existen documentos aproximadamente entre un periodo no mayor a quince días pendientes por facturar, desde el momento que el cliente recibe su servicio (Compañías de Seguro y Empresas) y en la gestión de cobranzas por parte del supervisor encargado del departamento, ya que la respuesta obtenida por el cliente al realizar dicha gestión arroja una evidencia en que las facturas son entregadas a destiempo luego de pasar una serie de días lo cual atrasa el sistema y la disponibilidad de la organización.

Ésta (factura) debe ser entregada con una serie de documentación llamada remesa, dichas operaciones se facturan quince días después de haber prestado el servicio, de igual manera, él envió de las facturas se realiza en un tiempo de cinco días después de emitir el documento fiscal.

En el mismo orden de ideas, se evidencia que no se realiza un control en el proceso de facturación, además no cuenta con normas y manuales de procedimientos por escritos, ni estrategias que les permita agilizar dicho proceso, lo cual afecta la operatividad de los departamentos ya que no son registradas oportunamente trayendo como consecuencia un 
desconocimiento contable en las operaciones administrativas de la empresa. Observándose una deficiencia en la planeación, organización, ejecución y comunicación entre los distintos departamentos de la empresa.

Por ende todo lo mencionado anteriormente, afecta a la gestión del proceso de cobranzas, la liquidez financiera. Es por ello, que se hace necesario diagnosticar los procedimientos aplicados a las actividades relacionadas con la facturación y cobranza para determinar sus reales limitaciones.

Con el objetivo de plantear alternativas que permitan corregir las debilidades, a través de un Plan de Mejoras al proceso de facturación para optimizar el Sistema de Cobranza de la Empresa Grupo Vitasana C.A.

\section{MÉTODO}

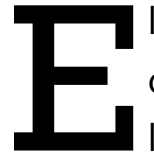
tipo de investigación de acuerdo a sus objetivos y las características de la misma fue cuantitativo, bajo la modalidad de proyecto factible ya que se orientó a dar una solución a la problemática planteada, a través de una propuesta basada en estrategias que permitan mejorar los procesos de facturación y cobranza en el Grupo Vitasana.

Apoyado, en un diseño de campo y documental, dado que los datos se recolectaron en el mismo lugar donde ocurrieron los hechos, en este caso la empresa Grupo Vitasana, permitiendo obtener información de primera mano sobre la situación que se presenta en el proceso de facturación y que afecta al sistema de cobranzas.

Documental, ya que se recurrió a antecedentes de investigaciones realizadas a lo que respecta a propuestas de mejoras en procesos de facturación, se citaron autores y se investigó en diferentes fuentes bibliográficas con el objetivo de definir lo planteado en los objetivos de la investigación.

En relaciona a la técnica y recolección de datos, en este caso se empleó la encuesta, utilizando como instrumento el cuestionario como medio principal para reunir información.

Para el desarrollo de la investigación la población de estudio es finita y estuvo conformada por 9 trabajadores del departamento de facturación y cobranza. En cuanto a la muestra en la presente investigación fue igual a la población ya que ésta es finita.

Confiabilidad para la determinación de la confiabilidad del instrumento, se realizó un estudio a la población objeto de estudio, posteriormente, los resultados fueron estudiados con el procedimiento de Medidas de Estabilidad al respecto Hernández y Otros (2004), señalan que "en este procedimiento un mismo instrumento de medición se aplica dos o más veces a un mismo grupo de personas, después de cierto período" (p. 365). 


\section{RESULTADOS}

$\mathrm{U}$ na vez analizada la situación actual en el área de facturación y cobranza Grupo Vitasana C.A se pudo evidenciar que no existe un adecuado control en el proceso de facturación, además no manejan normas y manuales de procedimientos por escritos, ni estrategias que les permita agilizar los proceso, lo que trae como consecuencia la infactivilidad de los departamentos, debido a que no son registradas oportunamente los servicios prestados, lo que conlleva a un desconocimiento contable en las operaciones administrativas de la empresa. Observándose una deficiencia en la planeación, organización, ejecución y comunicación entre los distintos departamentos de la empresa.

De acuerdo a lo antes señalado, se considera pertinente plantear un plan de mejoras en el proceso de facturación que optimice el sistema de cobranza de la empresa Grupo Vitasana, C.A., con el propósito satisfacer las necesidades de los clientes a través del ofrecimiento de un servicio excelente con condiciones crediticias, de calidad y servicio de pago, adicionalmente a ello la misma puede optimizar considerablemente el nivel de saldos en cartera, conjugando las variables de servicio, precio, créditos, días de pago u otros elementos. Por tal motivo, se propone un plan de mejoras al proceso de facturación, a través de métodos de trabajo que permitan la correcta facturación sin errores algunos y disponer el cobro a tiempo, para cumplir con los compromisos adquiridos.

Las alternativas para implementar en la empresa Grupo Vitasana, C.A. con el propósito de mejorar el proceso de facturación para optimizar el sistema de cobranza son las siguientes:

- Establecer los procedimientos a seguir para la facturación

- Establecer medidas en la gestión de cobro de las facturas

- Establecer políticas de Crédito que permitan la eficiencia en el Sistema de Cobranza

La primera alternativa, que consiste en establecer los procedimientos para la facturación es fundamental para el Grupo Vitasana ya que la factura es un documento de suma importancia y debe ser emitida con la mayor precisión, claridad y exactitud. Cualquier error en la confección de la factura supone un problema de cobro. Por tal motivo, la empresa debe contar con normas y procedimientos acordes que indiquen las instrucciones que deben seguirse para facturar el servicio prestado, de tal manera que se cumplan con las políticas administrativas y financieras de la empresa.

A continuación en el cuadro 1 se detalla el procedimiento de facturación: 
Cuadro 1. Procedimiento de facturación

\section{Responsable $\quad$ Actividad}

Jefe del Dpto. de Recibe el expediente médico de los pacientes y verifica que la documentación este Facturación acorde de acuerdo a las exigencias de cada seguro.

Si esta todo en orden lo envía al analista para proceder a facturarlo.

En caso de faltar algún documento el caso se envía al analista de admisión para que el anexe el documento faltante.

Este proceso se realizará en un máximo de dos días para aquellos casos que solo sean de consulta médica y laboratorios.

Y en caso de que sea consulta y algún tipo de examen (ecos, resonancia, citologías entre otros...) en un plazo no mayor a cinco días.

Envía relación de las facturas diarias al Dpto. de Cobranza.

Revisa todos los soportes de cada caso para proceder a emitir la factura.

Analista de Envía la Valija con las facturas a los Seguros respectivos en caso que sea una

Facturación factura personal deberá llamar al paciente para que la reciba.

Desglosa y distribuye las facturas original y copia con sus respectivos soportes.

Emite Relación de facturas diarias a su jefe inmediato.

La segunda alternativa, consiste en establecer medidas en la gestión de cobro las cuales serán:

- Llamar al cliente en un lapso de 5 días luego de la entrega de la factura para recordarle el pago de la misma.

- Enviar el estado de cuenta por correo semanalmente a los clientes.

- Si el seguro vence el plazo de los 45 días en cancelar sus facturas éste será suspendido hasta que haya cancelado el monto correspondiente.

- Calcular la rotación de las cuentas por cobrar mensualmente con la finalidad de ver si la gestión está siendo eficiente o no.

La tercera alternativa propuesta, consiste en establecer políticas de Crédito que permitan la eficiencia en el Sistema de Cobranza las cuales serían:

- Los montos y plazos de Crédito serán solamente autorizados por la Gerencia General

- La Gerencia General deberá mantener al día la información de cada uno de sus clientes actuales y potenciales a fin de conocer su capacidad de crédito, con el objeto de asegurar el cobro de los créditos que se le otorguen. 
- La ampliación de los plazos de Crédito se considerará únicamente para las empresas de Seguro que tengan una antigüedad mayor a cuatro años y hayan demostrado seriedad y puntualidad en sus pagos.

\section{CONCLUSIÓN}

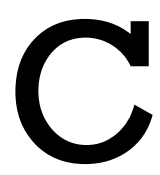

omo en cualquier proceso clave, la organización y el control toman importancia para que la gestión se realice correctamente. Y con el proceso de facturación no podía ser menos. En un negocio, gestionar correctamente la actividad operatividad y su facturación son elementos prioritarios. Sin embargo, todo puede quedar en nada si previamente no se establecen ciertos componentes organizativos que mejoren el proceso y lo hagan más eficiente.

Por lo tanto, la propuesta de un plan de mejoras en el proceso de facturación para optimizar el sistema de cobranza beneficiara a la empresa ya que al cumplir las normas y procedimientos establecidos le permitirá de una u otra forma a la organización tener un buen control, sobre todos los ingresos diarios que se obtenga en la misma, y de esa forma poder emitir una factura que permita englobar la documentación pertinente que se requiera para así agilizar su envió al destinatario en este caso (compañía de seguros, empresas), lo cual generara un buen sistema de cobranza en un tiempo real y oportuno.

Así mismo, para que la propuesta planteada pueda aplicarse y tenga éxito, se requiere la participación no solo de los empleados y gerentes de la empresa, sino también de los clientes a los cuales se les otorguen los días de créditos, los cuales deben aceptar las políticas de la empresa al momento de solicitar la prestación del servicio, y comprometerse a cumplir con los lapsos señalados para los pagos, para que no sean suspendido sus servicio y evitar molestias al paciente ya que es el primer afectado .

De igual forma, la empresa debe contar con procedimientos administrativos y contables para llevar a cabo las actividades de facturación, la cual no permitiría la deficiencia operativa en la cobranza.

Por otro lado, se debe tener en cuenta que la gestión de cobro es de vital importancia para cumplir con los compromisos de pago y proveer la operatividad del servicio.

A su vez, se deben realizar revisiones periódicas de los procedimientos de facturación como también de las cobranzas dado que no se programan acciones encaminadas a evitar el incumplimiento de pago por parte de los clientes y así disminuir el riesgo de mora de los mismos, la junta directiva podrá contar con un flujo de caja que le permita cubrir en gran parte sus obligaciones. 


\section{REFERENCIAS}

Harrigton, J. (2007). Mejoramientos de los Procesos de la Empresa. Mcgraw-Hill. Colonia Hernández; Fernández, y Batista. (2004). Metodología De la Investigación. Editorial Mc Graw Hill Editores. México

Hurtado, I., y Toro, J. (2006). Paradigmas y métodos de Investigación. Editorial Episteme, Venezuela
Terry y Franklin (2007). Principios de Administración. Edit. CECSA, $7^{\text {a }}$ reimpresión. México 